\title{
Control of Quantum Langevin Equations
}

\author{
Luigi Accardi \\ Centro Vito Volterra, Università di Roma Tor Vergata \\ Via Columbia, 2-00133 Roma, Italy \\ email: accardi@volterra.mat.uniroma2.it \\ Andreas Boukas* \\ Department of Mathematics, American College of Greece \\ Aghia Paraskevi, Athens 15342, Greece \\ email: gxk-personnel@ath.forthnet.gr
}

(Received: December 18, 2002)

\begin{abstract}
The problem of controlling quantum stochastic evolutions arises naturally in several different fields such as quantum chemistry, quantum information theory, quantum engineering, etc. In this paper, we apply the recently discovered closed form of the unitarity conditions for stochastic evolutions driven by the square of white noise [9] to solve this problem in the case of quadratic cost functionals (cf. (5.5) below). The optimal control is explicitly given in terms of the solution of an operator Riccati equation. Under general conditions on the system Hamiltonian part of the stochastic evolution and on the system observable to be controlled, this equation admits solutions with the required properties and they can be explicitly described.
\end{abstract}

\section{Introduction}

The problem of optimal control of solutions of a quantum Langevin equation with constant coefficients (see Definition 3 below) arises naturally in several different fields such as quantum chemistry, quantum information, quantum engineering, etc. The mathematical formulation of this problem was recently considered in $[8,10]$ for quantum systems affected by first order white noise. It was preceded by several studies on the quadratic control of the solution of a quantum evolution (see Definition 1 below) driven by first order white noise [24, 25, 26, 27] and the dual Kalman-Bucy filtering problem $[1,26]$. A general treatment of the control problem for quantum evolution driven by a general class of quantum noises can be found in [10] with the use of the representation free calculus of [12]. The quadratic form of the control criterion allows the quantum control problem to be solved in analogy with the classical stochastic control problem with the use of quantum stochastic calculus. The statement of the problem is the following: one starts from the Langevin equation for a system observable $X$ (cf. equations (4.3) and (5.4)

* The second author wishes to express his gratitude to Professor Luigi Accardi for his support and guidance as well as for the hospitality of the Centro Vito Volterra of the Universita di Roma TorVergata on several occasions over the past 15 years. 
below) and then looks for the coefficients ( $L, W$ for first order white noise and $D_{-}, W$ for the square of white noise) of the martingale terms of this equation, which minimize a given cost functional (cf. equations (4.4) and (5.5) below: in our case the functional is quadratic). Once this problem is solved, using the stochastic limit technique [15], one then looks for a bona fide Hamiltonian interaction which, in the stochastic limit, gives rise to the optimal Langevin equation.

Thus, combining techniques of quantum stochastic control with the stochastic limit of quantum theory, one can find a real physical interaction to concretely realize the optimal quantum evolution. In the classical case the optimal control is constructed via the solution of an operator Riccati differential equation. In the quantum case the equation is replaced by a quantum stochastic Riccati equation which in the problem considered in this paper is reduced to an operator algebraic Riccati equation (cf. equations (4.22) and (5.17)).

In Sec. 2 and 3 we define quantum evolutions, Langevin equations, the first order and the square of white noise stochastic differentials. In Sec. 4 and 5 we review the results on the control of first order white noise evolutions and Langevin equations (extended to include the conservation operator) and we solve the corresponding square of white noise control problem. For both first and second order quantum Langevin equations the theory of quadratic control should be extended to the case of noisy partial observations.

For the white noise approach to stochastic calculus, as well as for the square of white noise calculus we use primarily the results of $[9]$ and also $[3,4,5,6,7,11]$ and $[13,14,16,18,19,20,21,22,23,28]$. For more details on the technical aspects of first order quantum stochastic calculus we refer to [29].

\section{Quantum Evolutions and Langevin Equations}

DEFINITION 1 Let $a_{t}, a_{t}^{\dagger}$ be the standard Fock white noise of [15] and [16] defined on the Fock space $\Gamma$ over $L^{2}\left(\mathbb{R}_{+}, \mathbb{C}\right)$ in the case of first order white noise, while for the square of white noise it is over $L^{2}\left(\mathbb{R}_{+}, l_{2}(\mathbb{N})\right)$. For each $i=1,2,3, \ldots$ and $t \geq 0$ let

$$
N_{i}(t)=\int_{0}^{t} p_{i}\left(a_{s}, a_{s}^{\dagger}\right) d s
$$

be a noise process on $\Gamma$, where $p_{i}\left(a_{s}, a_{s}^{\dagger}\right)$ is a polynomial in $a_{s}$ and $a_{s}^{\dagger}$ written in normal order. Let also $\left\{C_{0}(t): t \geq 0\right\}$ and $\left\{C_{i}(t): t \geq 0\right\}$ be adapted bounded operator processes on the system Hilbert space $\mathcal{H}$. If

$$
h_{s}=C_{0}(s)+\sum_{i} C_{i}(s) p_{i}\left(a_{s}, a_{s}^{\dagger}\right),
$$

then

$$
H_{t}=\int_{0}^{t} h_{s} d s=\int_{0}^{t}\left(C_{0}(s)+\sum_{i} C_{i}(s) p_{i}\left(a_{s}, a_{s}^{\dagger}\right)\right) d s
$$




$$
=\int_{0}^{t} C_{0}(s) d s+\sum_{i} C_{i}(s) d N_{i}(s)
$$

is called a normally ordered white noise Hamiltonian on $\mathcal{H} \otimes \Gamma$. A unitary evolution is a family $U=\left\{U_{t}: t \geq 0\right\}$ of unitary operators on $\mathcal{H} \otimes \Gamma$ satisfying the normally ordered white noise Schrödinger equation

$$
\frac{d U_{t}}{d t}=-i h_{t} U_{t}, \quad U_{0}=1,
$$

and its adjoint

$$
\frac{d U_{t}^{*}}{d t}=i U_{t}^{*} h_{t}^{*}, \quad U_{0}^{*}=1,
$$

or, equivalently, the quantum stochastic differential equation

$$
d U_{t}=-i\left(d H_{t}\right) U_{t}, \quad U_{0}=1,
$$

and its adjoint

$$
d U_{t}^{*}=i U_{t}^{*}\left(d H_{t}^{*}\right), \quad U_{0}^{*}=1 .
$$

DEFINITION 2 Let $H$ and $U$ be as in Definition 1, let $X$ be an observable on the system Hilbert space $\mathcal{H}$, and let $\mathbb{1}$ denote the identity operator on $\Gamma$. A quantum flow is a family $j(X)=\left\{j_{t}(X)=U_{t}^{*}(X \otimes \mathbb{1}) U_{t}: t \geq 0\right\}$ of operators on $\mathcal{H} \otimes \Gamma$ satisfying the quantum Langevin equation

$$
\begin{aligned}
d j_{t}(X) & =d U_{t}^{*}(X \otimes \mathbb{1}) U_{t}+U_{t}^{*}(X \otimes \mathbb{1}) d U_{t}+d U_{t}^{*}(X \otimes \mathbb{1}) d U_{t} \\
& =i U_{t}^{*} h_{t}^{*}(X \otimes \mathbb{1}) U_{t} d t-i U_{t}^{*}(X \otimes \mathbb{1}) h_{t} U_{t} d t+U_{t}^{*} h_{t}^{*}(X \otimes \mathbb{1}) h_{t} U_{t}(d t)^{2} \\
& =i U_{t}^{*}\left(h_{t}^{*}(X \otimes \mathbb{1})-(X \otimes \mathbb{1}) h_{t}\right) U_{t} d t+U_{t}^{*} h_{t}^{*}(X \otimes \mathbb{1}) h_{t} U_{t}(d t)^{2} \\
& =j_{t}\left(i \rho\left(h_{t}, X \otimes \mathbb{1}\right)\right) d t+j_{t}\left(h_{t}^{*}(X \otimes \mathbb{1}) h_{t}\right)(d t)^{2},
\end{aligned}
$$

where $\rho(x, y):=x^{*} y-y x$ agrees with the usual commutator $[x, y]$ if $x^{*}=x$.

Equivalently, (2.7) can be written as

$$
d j_{t}(X)=j_{t}\left(i \rho\left(d H_{t}, X \otimes \mathbb{1}\right)\right) d t+j_{t}\left(d H_{t}^{*}(X \otimes \mathbb{1}) d H_{t}\right) .
$$

The $j_{t}\left(h_{t}^{*}(X \otimes \mathbb{1}) h_{t}\right)(d t)^{2}$ term in $(2.7)$ is computed with the use of the following rules (see $[17]$ ):

(a) Commute coefficient processes with the white noise functionals appearing in $h_{t}$.

(b) Put the result in normal order (i.e $a_{t}^{\dagger} a_{t}$ ) using the commutation rule $\left[a_{t}, a_{t}^{\dagger}\right]=$ $\delta(0)$.

(c) Treat $d t$ as a scalar.

(d) Replace the expression $\delta(0) d t$ by 1 .

(e) Replace the product of any normally ordered expression times $d t^{2}$ by 0 . 
In the case of the square of white noise, rules (a)-(e) lead to "renormalization" choices such as the subtraction of an infinite constant or the product of two delta functions. Both choices and their consequences have been extensively studied in $[4,5,6,7,9,11,14]$.

The computation of the $j_{t}\left(d H_{t}^{*}(X \otimes \mathbb{1}) d H_{t}\right)$ term in (2.8) by means of the above rules gives the same result that one obtains when this term is computed with the use of the Itô table for the stochastic differentials appearing in $d H_{t}$. In what follows we will use the formalism of (2.8).

\section{Quantum Stochastic Differentials}

Let $\mathcal{K}=\mathbb{C}$ in the case of first order white noise and $\mathcal{K}=l_{2}(\mathbb{N})$ for the square of white noise. The Boson Fock space $\Gamma=\Gamma\left(L^{2}\left(\mathbb{R}_{+}, \mathcal{K}\right)\right)$ can be defined as the Hilbert space completion of the linear span of the exponential vectors $\psi(f)$ under the inner product

$$
\langle\psi(f), \psi(g)\rangle=e^{\langle f, g\rangle}
$$

where $f, g \in L^{2}\left(\mathbb{R}_{+}, \mathcal{K}\right) \equiv L^{2}\left(\mathbb{R}_{+}\right) \otimes \mathcal{K}$. For $f \in L^{2}\left(\mathbb{R}_{+}, \mathcal{K}\right)$ and an adjointable linear operator $F$ on $L^{2}\left(\mathbb{R}_{+}, \mathcal{K}\right)$ the annihilation, creation and conservation operators $A(f), A^{\dagger}(f)$ and $\Lambda(F)$, respectively, are defined on the exponential vectors of $\Gamma$ by

$$
\begin{aligned}
A(f) \psi(g) & =\langle f, g\rangle \psi(g), \\
A^{\dagger}(f) \psi(g) & =\left.\frac{\partial}{\partial \epsilon}\right|_{\epsilon=0} \psi(g+\epsilon f), \\
\Lambda(F) \psi(g) & =\left.\frac{\partial}{\partial \epsilon}\right|_{\epsilon=0} \psi\left(e^{\epsilon F} g\right),
\end{aligned}
$$

where $F$ must be such that the exponential $e^{\epsilon F}$ is defined.

The first order quantum stochastic differentials $d A_{t}, d A_{t}^{\dagger}$, and $d \Lambda_{t}$ are defined by:

$$
\begin{aligned}
d A_{t}(f)=A\left(\chi_{[t, t+d t]} \otimes f\right), & f \in L^{2}\left(\mathbb{R}_{+}, \mathcal{K}\right), \\
d A_{t}^{\dagger}(f)=A^{\dagger}\left(\chi_{[t, t+d t]} \otimes f\right), & f \in L^{2}\left(\mathbb{R}_{+}, \mathcal{K}\right), \\
d \Lambda_{t}(F)=\Lambda\left(\chi_{[t, t+d t]} \otimes F\right), & F \in \mathcal{D}(\mathcal{K}),
\end{aligned}
$$

where $\mathcal{D}(\mathcal{K})$ is the space of adjointable operators on $\mathcal{K}$.

Notice that $\chi_{[t, t+d t]}$ is a vector in $A^{+}(\cdot), A(\cdot)$ and a multiplication operator in $\Lambda(\cdot)$. The multiplication rules for the stochastic differentials are given in the following:

PROPOSITION 1 The first order white noise Itô table is (see [29]) 


\begin{tabular}{c|cccc} 
& $d A_{t}^{\dagger}\left(f_{1}\right)$ & $d \Lambda_{t}\left(F_{1}\right)$ & $d A_{t}\left(f_{1}\right)$ & $d t$ \\
\hline$d A_{t}^{\dagger}\left(f_{2}\right)$ & 0 & 0 & 0 & 0 \\
$d \Lambda_{t}\left(F_{2}\right)$ & $d A_{t}^{\dagger}\left(F_{2} f_{1}\right)$ & $d \Lambda_{t}\left(F_{2} F_{1}\right)$ & 0 & 0 \\
$d A_{t}\left(f_{2}\right)$ & $\left\langle f_{2}, f_{1}\right\rangle d t$ & $d A_{t}\left(F_{1}^{*} f_{2}\right)$ & 0 & 0 \\
$d t$ & 0 & 0 & 0 & 0
\end{tabular}

The square of white noise quantum stochastic differentials $d M_{t}, d B_{t}^{+}$, and $d B_{t}^{-}$ are defined by

$$
\begin{aligned}
d M_{t} & =d \Lambda_{t}\left(\rho^{+}(M)\right)+d t, \\
d B_{t}^{+} & =d \Lambda_{t}\left(\rho^{+}\left(B^{+}\right)\right)+d A_{t}^{\dagger}\left(e_{0}\right), \\
d B_{t}^{-} & =d \Lambda_{t}\left(\rho^{+}\left(B^{-}\right)\right)+d A_{t}\left(e_{0}\right),
\end{aligned}
$$

where for $n, k, l, m \in\{0,1, \ldots\}$

$$
\begin{aligned}
d \Lambda_{n, k, l}(t) & =d \Lambda_{t}\left(\rho^{+}\left(B^{+n} M^{k} B^{-l}\right)\right), \\
d A_{m}(t) & =d A_{t}\left(e_{m}\right), \\
d A_{m}^{\dagger}(t) & =d A_{t}^{\dagger}\left(e_{m}\right),
\end{aligned}
$$

where we have used the notation

$$
d X_{t}(y)=X\left(\chi_{[t, t+d t]} \otimes y\right)
$$

and the operators $\rho^{+}\left(B^{+n} M^{k} B^{-k}\right)$ are defined by

$$
\rho^{+}\left(B^{+n} M^{k} B^{-l}\right) e_{m}=\theta_{n, k, l, m} e_{n+m-l},
$$

where $e_{m}, m=0,1,2, \ldots$ is any orthonormal basis of $l_{2}(\mathbb{N})$,

$$
\theta_{n, k, l, m}:=H(n+m-l) \sqrt{\frac{m-l+n+1}{m+1}} 2^{k}(m-l+1)_{n}(m+1)^{(l)}(m-l+1)^{k},
$$

$H(x)$ is the Heaviside function $(H(x)=0$ for $x<0$; $H(x)=1$ for $x \geq 0)$,

$$
0^{0}=1, \quad\left(B^{+}\right)^{n}=\left(B^{-}\right)^{n}=N^{n}=0, \quad \text { for } \quad n<0,
$$

and the "factorial powers" are defined by

$$
\begin{aligned}
& x^{(n)}=x(x-1) \cdots(x-n+1), \\
& (x)_{n}=x(x+1) \cdots(x+n-1), \\
& (x)_{0}=x^{(0)}=1 .
\end{aligned}
$$

The Itô multiplication table for $d \Lambda_{n, k, l}(t), d A_{m}(t)$, and $d A_{m}^{\dagger}(t)$ is

$$
\begin{aligned}
d \Lambda_{\alpha, \beta, \gamma}(t) d \Lambda_{a, b, c}(t) & =\sum c_{\beta, \gamma, a, b}^{\lambda, \rho, \sigma, \omega, \epsilon} d \Lambda_{a+\alpha-\gamma+\lambda, \omega+\sigma+\epsilon, \lambda+c}(t) \\
d \Lambda_{\alpha, \beta, \gamma}(t) d A_{n}^{\dagger}(t) & =\theta_{\alpha, \beta, \gamma, n} d A_{\alpha+n-\gamma}^{\dagger}(t) \\
d A_{m}(t) d \Lambda_{a, b, c}(t) & =\theta_{c, b, a, m} d A_{c+m-a}(t) \\
d A_{m}(t) d A_{n}^{\dagger}(t) & =\delta_{m, n} d t
\end{aligned}
$$


where

$$
\begin{aligned}
& c_{\beta, \gamma, a, b}^{\lambda, \rho, \sigma, \omega, \epsilon}= \\
& \left(\begin{array}{c}
\gamma \\
\lambda
\end{array}\right)\left(\begin{array}{c}
\gamma-\lambda \\
\rho
\end{array}\right)\left(\begin{array}{c}
\beta \\
\omega
\end{array}\right)\left(\begin{array}{l}
b \\
\epsilon
\end{array}\right) 2^{\beta+b-\omega-\epsilon} S_{\gamma-\lambda-\rho, \sigma} a^{(\gamma-\lambda)}(a+\lambda-1)^{(\rho)}(a-\gamma+\lambda)^{\beta-\omega} \lambda^{b-\epsilon},
\end{aligned}
$$

$S_{\gamma-\lambda-\rho, \sigma}$ are the Stirling numbers of the first kind and $\sum$ in (3.17) denotes the finite sum

$$
\sum_{\lambda=0}^{\gamma} \sum_{\rho=0}^{\gamma-\lambda} \sum_{\sigma=0}^{\gamma-\lambda-\rho} \sum_{\omega=0}^{\beta} \sum_{\epsilon=0}^{b}
$$

All other products of differentials are equal to zero.

To obtain a concise formulation of the square of white noise evolutions we proceed as follows. Let $\mathcal{D}(\mathcal{K})$ and $\mathcal{B}(\mathcal{H})$ denote, respectively, the spaces of adjointable operators on $\mathcal{K}$ and bounded linear operators on $\mathcal{H}$. The tensor product $\mathcal{B}(\mathcal{H}) \otimes \mathcal{K}$ is a pre-Hilbert module with $\mathcal{B}(\mathcal{H})$-valued inner product defined on elementary tensors by

$$
(a \otimes \xi \mid b \otimes \eta)=a^{*} b\langle\xi, \eta\rangle .
$$

On $\mathcal{B}(\mathcal{H}) \otimes \mathcal{K}$ define linear operators $\mathcal{A}$ and $\mathcal{A}^{\dagger}$ by

$$
\begin{aligned}
\mathcal{A}(a \otimes \xi) & =a \otimes A(\xi), \\
\mathcal{A}^{\dagger}(a \otimes \xi) & =a \otimes A^{\dagger}(\xi),
\end{aligned}
$$

while on $\mathcal{B}(\mathcal{H}) \otimes \mathcal{D}(\mathcal{K})$ define a linear operator $\mathcal{L}$ by

$$
\mathcal{L}(a \otimes T)=a \otimes \Lambda(T) .
$$

For $\alpha, \beta, \gamma, a, b, c \in\{0,1,2, \ldots\}$, if $\left\{D_{\alpha, \beta, \gamma}\right\}$ and $\left\{E_{a, b, c}\right\}$ are families of operators in $\mathcal{B}(\mathcal{H})$ and

$$
D=\sum_{\alpha, \beta, \gamma} D_{\alpha, \beta, \gamma} \otimes \rho^{+}\left(B^{+^{\alpha}} M^{\beta} B^{-\gamma}\right), \quad E=\sum_{a, b, c} E_{a, b, c} \otimes \rho^{+}\left(B^{+a} M^{b} B^{-c}\right),
$$

define the o-product $D \circ E$ of $D$ and $E$ by

$$
D \circ E=\sum_{\alpha, \beta, \gamma} \sum_{a, b, c} \sum c_{\beta, \gamma, a, b}^{\lambda, \rho, \sigma, \omega, \epsilon} D_{\alpha, \beta, \gamma} E_{a, b, c} \otimes \rho^{+}\left(B^{+a+\alpha-\gamma+\lambda} M^{\omega+\sigma+\epsilon} B^{-\lambda+\varphi_{3 ., 26)}}\right.
$$

where $\sum$ and $c_{\beta, \gamma, a, b}^{\lambda, \rho, \sigma, \omega}$ are as in (3.17) and (3.21). Define also linear operators $r$ and $l$ on $\mathcal{B}(\mathcal{H}) \otimes \mathcal{D}(\mathcal{K})$ with values in the space of linear operators on $\mathcal{B}(\mathcal{H}) \otimes \mathcal{K}$ by

$$
\begin{aligned}
r(D) T & =\sum_{n, \alpha, \beta, \gamma} D_{\alpha, \beta, \gamma} \theta_{\alpha, \beta, \gamma, n-\alpha+\gamma} T_{n-\alpha+\gamma} \otimes e_{n}, \\
l(D) T & =\sum_{n, \alpha, \beta, \gamma} T_{n+\alpha-\gamma} \theta_{\gamma, \beta, \alpha, n+\alpha-\gamma} D_{\alpha, \beta, \gamma} \otimes e_{n},
\end{aligned}
$$


where $T=\sum_{n} T_{n} \otimes e_{n} \in \mathcal{B}(\mathcal{H}) \otimes \mathcal{K}, n \in\{0,1, \ldots\}$ and $\theta$ is as in (3.16). If

$$
\begin{aligned}
& D_{+}=\sum_{n} D_{+, n} \otimes e_{n}, \\
& D_{-}=\sum_{m} D_{-, m} \otimes e_{m}, \\
& D_{1}=\sum_{\alpha, \beta, \gamma} D_{1, \alpha, \beta, \gamma} \otimes \rho^{+}\left(B^{+\alpha} M^{\beta} B^{-\gamma}\right), \\
& E_{1}=\sum_{a, b, c} E_{1, a, b, c} \otimes \rho^{+}\left(B^{+a} M^{b} B^{-c}\right),
\end{aligned}
$$

where $n, m, \alpha, \beta, \gamma, a, b, c \in\{0,1,2, \ldots\}$ and $D_{+, n}, D_{-, m}, D_{1, \alpha, \beta, \gamma}, E_{1, a, b, c} \in \mathcal{B}(\mathcal{H})$, then

$$
\left(D_{-}^{*} \mid D_{+}\right)=\sum_{m, n} D_{-, m} D_{+, n}\left\langle e_{m}, e_{n}\right\rangle=\sum_{n} D_{-, n} D_{+, n}
$$

and the square of white noise Itô table takes the following form:

PROPOSITION 2 The square of white noise Itô table is (see [9]):

$$
\begin{aligned}
d \mathcal{A}_{t}\left(D_{-}\right) d \mathcal{A}_{t}^{\dagger}\left(D_{+}\right) & =\left(D_{-}^{*} \mid D_{+}\right) d t \\
d \mathcal{L}_{t}\left(D_{1}\right) d \mathcal{L}_{t}\left(E_{1}\right) & =d \mathcal{L}_{t}\left(D_{1} \circ E_{1}\right) \\
d \mathcal{L}_{t}\left(D_{1}\right) d \mathcal{A}_{t}^{\dagger}\left(D_{+}\right) & =d \mathcal{A}_{t}^{\dagger}\left(r\left(D_{1}\right) D_{+}\right) \\
d \mathcal{A}_{t}\left(D_{-}\right) d \mathcal{L}_{t}\left(E_{1}\right) & =d \mathcal{A}_{t}\left(l\left(E_{1}\right) D_{-}\right)
\end{aligned}
$$

All other products of stochastic differentials (including $d t$ ) are equal to zero.

The differential form of the square of white noise Hamiltonian operator is

$$
d H_{t}=D_{0}(t) d t+d \mathcal{L}_{t}\left(D_{1}\right)+d \mathcal{A}_{t}^{\dagger}\left(D_{+}\right)+d \mathcal{A}_{t}\left(D_{-}\right) .
$$

\section{Control of First Order White Noise Langevin Flows}

In the notation of Section 2 and 3 , we consider a quantum flow $\left\{j_{t}(X) / t \in[0, T]\right\}$ of bounded linear operators on $\mathcal{H} \otimes \Gamma$ defined by $j_{t}(X)=U_{t}^{*} X U_{t}$, where $\mathcal{H}$ is a separable Hilbert space, $\Gamma$ is the Boson Fock space over $L^{2}\left(\mathbb{R}_{+}, \mathbb{C}\right), X$ is a self-adjoint operator on $\mathcal{H}$ identified with its ampliation $X \otimes \mathbb{1}$ to $\mathcal{H} \otimes \Gamma$, and $U=\left\{U_{t}: t \geq 0\right\}$ is a unitary evolution satisfying on $\mathcal{H} \otimes \Gamma$ a quantum stochastic differential equation of the form

$$
d U_{t}=-\left(\left(i H+\frac{1}{2} L^{*} L\right) d t+L^{*} W d A_{t}-L d A_{t}^{\dagger}+(1-W) d \Lambda_{t}\right) U_{t}, t \in[0, T],
$$

with adjoint

$$
d U_{t}^{*}=-U_{t}^{*}\left(\left(-i H+\frac{1}{2} L^{*} L\right) d t-L^{*} d A_{t}+W^{*} L d A_{t}^{\dagger}+\left(1-W^{*}\right) d \Lambda_{t}\right), t \in[0, T]
$$


and initial conditions

$$
U_{0}=U_{0}^{*}=1,
$$

where $H, L, W$ are bounded operators on $\mathcal{H}$ with $H$ self-adjoint and $W$ unitary. These conditions guarantee the existence uniqueness and unitarity of the solution of (4.1), (4.2).

Using the Itô table for first order white noise we can show that the flow $\left\{j_{t}(X)\right.$ : $t \in[0, T]\}$ satisfies the quantum stochastic differential equation

$$
\begin{aligned}
d j_{t}(X)= & j_{t}\left(i[H, X]-\frac{1}{2}\left(L^{*} L X+X L^{*} L-2 L^{*} X L\right)\right) d t \\
& +j_{t}\left(\left[L^{*}, X\right] W\right) d A_{t}+j_{t}\left(W^{*}[X, L]\right) d A_{t}^{\dagger}++j_{t}\left(W^{*} X W-X\right) d \Lambda_{t}
\end{aligned}
$$

with initial condition

$$
j_{0}(X)=X, \quad t \in[0, T] .
$$

DEFINITION 3 On a finite time interval $[0, T]$, the cost functional for the solution of the quantum Langevin equation (4.3) is given by:

$$
J_{\xi, T}(L, W)=\int_{0}^{T}\left[\left\|j_{t}(X) \xi\right\|^{2}+\frac{1}{4}\left\|j_{t}\left(L^{*} L\right) \xi\right\|^{2}\right] d t+\frac{1}{2}\left\|j_{T}(L) \xi\right\|^{2},
$$

where $\xi$ is an arbitrary vector in the exponential domain of $\mathcal{H} \otimes \Gamma$.

Thinking of $L$ and $W$ as controls we interpret the first term of the right hand side of (4.4) as a measure of the size of the flow over $[0, T]$, the second as a measure of the control effort over $[0, T]$ and the third as a "penalty" for allowing the evolution to go on for a long time. We consider the problem of controlling the size of such a flow by minimizing the cost functional $J_{\xi, T}(L, W)$ of (4.4).

THEOREM A Let $U=\left\{U_{t}: t \geq 0\right\}$ be a process satisfying the quantum stochastic differential equation

$$
d U_{t}=\left(F U_{t}+u_{t}\right) d t+\Psi U_{t} d A_{t}+\Phi U_{t} d A_{t}^{\dagger}+Z U_{t} d \Lambda_{t}, \quad U_{0}=\mathbb{1}, \quad t \in[0, T],
$$

with adjoint

$d U_{t}^{*}=\left(U_{t}^{*} F^{*}+u_{t}^{*}\right) d t+U_{t}^{*} \Psi^{*} d A_{t}^{\dagger}+U_{t}^{*} \Phi^{*} d A_{t}+U_{t}^{*} Z^{*} d \Lambda_{t}, \quad U_{0}^{*}=\mathbb{1}, \quad t \in[0, T]$,

where $T>0$ is a fixed finite horizon, the coefficients $F, \Psi, \Phi, Z$ are bounded operators on the system space $\mathcal{H}$ and $u_{t}$ is of the form $-\Pi U_{t}$ for some positive bounded system operator $\Pi$.

Then the functional

$$
Q_{\xi, T}(u)=\int_{0}^{T}\left[\left\langle U_{t} \xi, X^{2} U_{t} \xi\right\rangle+\left\langle u_{t} \xi, u_{t} \xi\right\rangle\right] d t-\left\langle u_{T} \xi, U_{T} \xi\right\rangle
$$


where $X$ is a system space observable, identified with its ampliation $X \otimes I$ to $\mathcal{H} \otimes \Gamma$, is minimized over the set of feedback control processes of the form $u_{t}=-\Pi U_{t}$, by choosing $\Pi$ to be a bounded, positive, self-adjoint system operator satisfying

$$
\begin{aligned}
\Pi F+F^{*} \Pi+\Phi^{*} \Pi \Phi-\Pi^{2}+X^{2} & =0, \\
\Pi \Psi+\Phi^{*} \Pi+\Phi^{*} \Pi Z & =0 \\
\Pi Z+Z^{*} \Pi+Z^{*} \Pi Z & =0 .
\end{aligned}
$$

The minimum value is $\langle\xi, \Pi \xi\rangle$. We recognize (4.8) as the algebraic Riccati equation.

Proof. Let

$$
\theta_{t}=\left\langle\xi, U_{t}^{*} \Pi U_{t} \xi\right\rangle
$$

Using the identity $d(x y)=x d y+d x y+d x d y$ we obtain

$$
d \theta_{t}=\left\langle\xi, d\left(U_{t}^{*} \Pi U_{t}\right) \xi\right\rangle=\left\langle\xi,\left(d U_{t}^{*} \Pi U_{t}+U_{t}^{*} \Pi d U_{t}+d U_{t}^{*} \Pi d U_{t}\right) \xi\right\rangle,
$$

which, after replacing $d U_{t}$ and $d U_{t}^{*}$ by (4.5) and (4.6) respectively and using the Itô table of Proposition 1, becomes

$$
\begin{aligned}
d \theta_{t}= & \left\langle\xi, U_{t}^{*}\left(\left(F^{*} \Pi+\Pi F+\Phi^{*} \Pi \Phi\right) d t+\left(\Phi^{*} \Pi+\Pi \Psi+\Phi^{*} \Pi Z\right) d A_{t}\right.\right. \\
& \left.\left.+\left(\Psi \Pi^{*}+\Pi \Phi+Z^{*} \Pi \Phi\right) d A_{t}^{\dagger}+\left(Z^{*} \Pi+\Pi Z+Z^{*} \Pi Z\right) d \Lambda_{t}\right) U_{t} \xi\right\rangle \\
& +\left\langle\xi,\left(u_{t}^{*} \Pi U_{t}+U_{t}^{*} \Pi u_{t}\right) d t \xi\right\rangle,
\end{aligned}
$$

and by (4.8)-(4.10)

$$
d \theta_{t}=\left\langle\xi, U_{t}^{*}\left(\Pi^{2}-X^{2}\right) U_{t} d t \xi\right\rangle+\left\langle\xi,\left(u_{t}^{*} \Pi U_{t}+U_{t}^{*} \Pi u_{t}\right) d t \xi\right\rangle .
$$

By (4.11)

$$
\theta_{T}-\theta_{0}=\left\langle\xi, U_{T}^{*} \Pi U_{T} \xi\right\rangle-\langle\xi, \Pi \xi\rangle
$$

while by (4.14)

$$
\theta_{T}-\theta_{0}=\int_{0}^{T}\left(\left\langle\xi, U_{t}^{*}\left(\Pi^{2}-X^{2}\right) U_{t} \xi\right\rangle+\left\langle\xi,\left(u_{t}^{*} \Pi U_{t}+U_{t}^{*} \Pi u_{t}\right) \xi\right\rangle\right) d t
$$

By (4.15) and (4.16)

$$
\begin{aligned}
\left\langle\xi, U_{T}^{*} \Pi U_{T} \xi\right\rangle-\langle\xi, \Pi \xi\rangle= & \\
& \int_{0}^{T}\left(\left\langle\xi, U_{t}^{*}\left(\Pi^{2}-X^{2}\right) U_{t} \xi\right\rangle+\left\langle\xi,\left(u_{t}^{*} \Pi U_{t}+U_{t}^{*} \Pi u_{t}\right) \xi\right\rangle\right) d t .
\end{aligned}
$$

Thus

$$
Q_{\xi, T}(u)=\left(\left\langle\xi, U_{T}^{*} \Pi U_{T} \xi\right\rangle-\langle\xi, \Pi \xi\rangle\right)+Q_{\xi, T}(u)-\left(\left\langle\xi, U_{T}^{*} \Pi U_{T} \xi\right\rangle-\langle\xi, \Pi \xi\rangle\right) .
$$


Replacing the first parenthesis on the right hand side of (4.18) by (4.17), and $Q_{\xi, T}(u)$ by (4.7) we obtain after cancellations

$$
\begin{aligned}
Q_{\xi, T}(u) & =\int_{0}^{T}\left\langle\xi,\left(U_{t}^{*} \Pi^{2} U_{t}+u_{t}^{*} \Pi U_{t}+U_{t}^{*} \Pi u_{t}+u_{t}^{*} u_{t}\right) \xi\right\rangle d t+\langle\xi, \Pi \xi\rangle \\
& =\int_{0}^{T}\left\|\left(u_{t}+\Pi U_{t}\right) \xi\right\|^{2} d t+\langle\xi, \Pi \xi\rangle,
\end{aligned}
$$

which is clearly minimized by $u_{t}=-\Pi U_{t}$ and the minimum is $\langle\xi, \Pi \xi\rangle$.

DEFINITION 4 The pair $(i H, X)$ is called stabilizable if there exists a bounded system operator $K$ such that $i H+K X$ is the generator of an asymptotically stable semigroup $\mathcal{F}_{t}$ i.e there exist constants $M>0$ and $\omega<0$ such that $\left\|\mathcal{F}_{t}\right\| \leq M e^{\omega t}$ (see $[30])$.

THEOREM B Let $X$ be a bounded self-adjoint system operator such that the pair $(i H, X)$ is stabilizable. The quadratic performance functional (4.4) associated with the quantum stochastic flow $\left\{j_{t}(X)=U_{t}^{*} X U_{t}: t \geq 0\right\}$, where $U=\left\{U_{t}: t \geq 0\right\}$ is the solution of (4.1), is minimized by

$$
L=\sqrt{2} \Pi^{1 / 2} W_{1} \quad(\text { polar decomposition of } L)
$$

and

$$
W=W_{2},
$$

where $\Pi$ is a positive self-adjoint solution of the "algebraic Riccati equation"

$$
i[H, \Pi]+\Pi^{2}+X^{2}=0,
$$

and $W_{1}, W_{2}$ are bounded unitary system operators commuting with $\Pi$. Moreover

$$
\min _{L, W} J_{\xi, T}(L, W)=\langle\xi, \Pi \xi\rangle
$$

independently of $T$.

Remark 1 Eq. (4.22) is a special case of the algebraic Riccati equation (ARE). It is known (see [30]) that if the pair $(i H, X)$ is stabilizable, then (4.22) has a positive self-adjoint solution $\Pi$.

Proof. Looking at (4.1) as (4.5) with $u_{t}=-\frac{1}{2} L^{*} L U_{t}, F=-i H, \Psi=-L^{*} W$, $\Phi=L$, and $Z=W-1,(4.7)$ is identical to (4.4). Moreover, equations (4.8)-(4.10) become

$$
\begin{aligned}
i[H, \Pi]+L^{*} \Pi L-\Pi^{2}+X^{2} & =0, \\
L^{*} \Pi-\Pi L^{*} W+L^{*} \Pi(W-1) & =0, \\
\left(W^{*}-1\right) \Pi+\Pi(W-1)+\left(W^{*}-1\right) \Pi(W-1) & =0 .
\end{aligned}
$$


By the self-adjointness of $\Pi,(4.25)$ implies that

$$
[L, \Pi]=\left[L^{*}, \Pi\right]=0,
$$

while (4.26) implies that

$$
[W, \Pi]=\left[W^{*}, \Pi\right]=0,
$$

i.e (4.21). By (4.27) and the fact that in this case

$$
\Pi=\frac{1}{2} L^{*} L \quad \text { i.e } \quad L^{*} L=2 \Pi,
$$

eq. (4.24) implies (4.22). Eqs (4.27) and (4.29) also imply that

$$
\left[L, L^{*}\right]=0 \quad \text { (i.e } L \text { is normal) }
$$

which implies (4.20).

\section{Control of Square of White Noise Langevin Flows}

As shown in [9], eqs. (4.1) and (4.2) are replaced, respectively, by

$d U_{t}=\left(\left(-\frac{1}{2}\left(D_{-}^{*} \mid D_{-}^{*}\right)+i H\right) d t+d \mathcal{A}_{t}\left(D_{-}\right)+d \mathcal{A}_{t}^{\dagger}\left(-r(W) D_{-}^{*}\right)+d \mathcal{L}_{t}(W-I)\right) U_{t}$

and

$d U_{t}^{*}=U_{t}^{*}\left(\left(-\frac{1}{2}\left(D_{-}^{*} \mid D_{-}^{*}\right)-i H\right) d t+d \mathcal{A}_{t}^{\dagger}\left(D_{-}^{*}\right)+d \mathcal{A}_{t}\left(-l\left(W^{*}\right) D_{-}\right)+d \mathcal{L}_{t}\left(W^{*}-I\right)\right)$

with initial conditions

$$
U_{0}=U_{0}^{*}=\mathbb{1},
$$

where $H$ is any bounded self-adjoint system operator, $W$ is a o-product unitary operator such that $r(W) r\left(W^{*}\right)=r\left(W^{*}\right) r(W)=\mathbb{1}, I$ is the o-product identity, $D_{-}$ is an arbitrary operator as in (3.30), and $\mathbb{1}$ is the identity operator on $\mathcal{H} \otimes \Gamma$. These conditions guarantee the existence, uniqueness and unitarity of the solutions.

PROPOSITION 3 In the case of the square of white noise, the quantum Langevin equation (4.3) is replaced by

$$
\begin{aligned}
d j_{t}(X)=j_{t} & \left(i[X, H]-\frac{1}{2}\left(\left(D_{-}^{*} \mid D_{-}^{*}\right) X+X\left(D_{-}^{*} \mid D_{-}^{*}\right)\right)+\left(r(W) D_{-}^{*} \mid X r(W) D_{-}^{*}\right)\right) d t \\
& +j_{t}\left(d \mathcal{A}_{t}^{\dagger}\left(D_{-}^{*} X-r\left(W^{*} X\right) r(W) D_{-}^{*}\right)\right) \\
& +j_{t}\left(d \mathcal{A}_{t}\left(X D_{-}-l(X W) l\left(W^{*}\right) D_{-}\right)\right) \\
& +j_{t}\left(d \mathcal{L}_{t}\left(W^{*} X \circ W-X\right)\right)
\end{aligned}
$$


Proof.

$$
\begin{aligned}
& d j_{t}(X)=\left(d U_{t}^{*}\right) X U_{t}+U_{t}^{*} X\left(d U_{t}\right)+\left(d U_{t}^{*}\right) X\left(d U_{t}\right) \\
& =U_{t}^{*}\left\{\left(-\frac{1}{2}\left(D_{-}^{*} \mid D_{-}^{*}\right)-i H\right) X d t+d \mathcal{A}_{t}^{\dagger}\left(D_{-}^{*} X\right)-d \mathcal{A}_{t}\left(l\left(W^{*}\right) D_{-} X\right)\right. \\
& \left.+d \mathcal{L}_{t}\left(\left(W^{*}-I\right) X\right)\right)+X\left(-\frac{1}{2}\left(D_{-}^{*} \mid D_{-}^{*}\right)+i H\right) d t-d \mathcal{A}_{t}^{\dagger}\left(X r(W) D_{-}^{*}\right) \\
& +d \mathcal{A}_{t}\left(X D_{-}\right)+d \mathcal{L}_{t}(X(W-I))+\left(r(W) D_{-}^{*} \mid X r(W) D_{-}^{*}\right) d t \\
& -d \mathcal{A}_{t}\left(X l((W-I)) l\left(W^{*}\right) D_{-}\right)-d \mathcal{A}_{t}^{\dagger}\left(r\left(\left(W^{*}-I\right) X\right) r(W) D_{-}^{*}\right) \\
& \left.+d \mathcal{L}_{t}\left(\left(W^{*}-I\right) X \circ(W-I)\right)\right\} U_{t} \\
& =U_{t}^{*}\left\{\left(-\frac{1}{2}\left(\left(D_{-}^{*} \mid D_{-}^{*}\right) X+X\left(D_{-}^{*} \mid D_{-}^{*}\right)\right)+i[X, H]\right.\right. \\
& \left.+\left(r(W) D_{-}^{*} \mid X r(W) D_{-}^{*}\right)\right) d t+d \mathcal{A}_{t}^{\dagger}\left(D_{-}^{*} X-X r(W) D_{-}^{*}\right. \\
& \left.-r\left(\left(W^{*}-I\right) X\right) r(W) D_{-}^{*}\right)-d \mathcal{A}_{t}\left(l\left(W^{*}\right) D_{-} X-X D_{-}\right. \\
& \left.+l(X(W-I)) l\left(W^{*}\right) D_{-}\right)+d \mathcal{L}_{t}\left(\left(W^{*}-I\right) X+X(W-I)\right. \\
& \left.\left.+\left(W^{*}-I\right) X \circ(W-I)\right)\right\} U_{t} \\
& =U_{t}^{*}\left\{-\frac{1}{2}\left(\left(D_{-}^{*} \mid D_{-}^{*}\right) X+X\left(D_{-}^{*} \mid D_{-}^{*}\right)\right)+i[X, H]\right. \\
& \left.+\left(r(W) D_{-}^{*} \mid X r(W) D_{-}^{*}\right)\right\} U_{t} d t \\
& +U_{t}^{*}\left\{d \mathcal{A}_{t}^{\dagger}\left(D_{-}^{*} X-X r(W) D_{-}^{*}-r\left(\left(W^{*}-I\right) X\right) r(W) D_{-}^{*}\right)\right\} U_{t} \\
& +U_{t}^{*}\left\{d \mathcal{A}_{t}\left(-l\left(W^{*}\right) D_{-} X+X D_{-}-l(X(W-I)) l\left(W^{*}\right) D_{-}\right)\right\} U_{t} \\
& +U_{t}^{*}\left\{d \mathcal{L}_{t}\left(W^{*} X \circ W-X\right)\right\} U_{t} \\
& =j_{t}\left(i[X, H]-\frac{1}{2}\left(\left(D_{-}^{*} \mid D_{-}^{*}\right) X+X\left(D_{-}^{*} \mid D_{-}^{*}\right)\right)+\left(r(W) D_{-}^{*} \mid X r(W) D_{-}^{*}\right)\right) d t \\
& +j_{t}\left(d \mathcal{A}_{t}^{\dagger}\left(D_{-}^{*} X-X r(W) D_{-}^{*}-r\left(\left(W^{*}-I\right) X\right) r(W) D_{-}^{*}\right)\right) \\
& +j_{t}\left(d \mathcal{A}_{t}\left(X D_{-}-l\left(W^{*}\right) D_{-} X-l(X(W-I)) l\left(W^{*}\right) D_{-}\right)\right) \\
& +j_{t}\left(d \mathcal{L}_{t}\left(W^{*} X \circ W-X\right)\right) \\
& =j_{t}\left(i[X, H]-\frac{1}{2}\left(\left(D_{-}^{*} \mid D_{-}^{*}\right) X+X\left(D_{-}^{*} \mid D_{-}^{*}\right)\right)\right. \\
& \left.+\left(r(W) D_{-}^{*} \mid X r(W) D_{-}^{*}\right)\right) d t \\
& +j_{t}\left(d \mathcal{A}_{t}^{\dagger}\left(D_{-}^{*} X-r\left(W^{*} X\right) r(W) D_{-}\right)\right) \\
& +j_{t}\left(d \mathcal{A}_{t}\left(X D_{-}-l(X W) l\left(W^{*}\right) D_{-}\right)\right) \\
& +j_{t}\left(d \mathcal{L}_{t}\left(W^{*} X \circ W-X\right)\right) \text {. }
\end{aligned}
$$

DEFINITION 5 On a finite time interval $[0, T]$, the cost functional for the solution 
of the quantum Langevin equation (5.4) is given by:

$J_{\xi, T}\left(D_{-}, W\right)=\int_{0}^{T}\left[\left\|j_{t}(X) \xi\right\|^{2}+\frac{1}{4}\left\|j_{t}\left(\left(D_{-}^{*} \mid D_{-}^{*}\right)\right) \xi\right\|^{2}\right] d t+\frac{1}{2}\left\langle\xi, j_{T}\left(\left(D_{-}^{*} \mid D_{-}^{*}\right)\right) \xi\right\rangle$,

where $\xi$ is an arbitrary vector in the exponential domain of $\mathcal{H} \otimes \Gamma$.

The square of white noise analogues of Theorems 1 and 2 are as follows.

THEOREM C Let $U=\left\{U_{t}: t \geq 0\right\}$ be a process satisfying the quantum stochastic differential equation

$$
d U_{t}=\left(F U_{t}+u_{t}\right) d t+d \mathcal{A}_{t}(\Psi) U_{t}+d \mathcal{A}_{t}^{\dagger}(\Phi) U_{t}+d \mathcal{L}_{t}(Z) U_{t}, U_{0}=\mathbb{1}, t \in[0, T],
$$

with adjoint

$d U_{t}^{*}=\left(U_{t}^{*} F^{*}+u_{t}^{*}\right) d t+U_{t}^{*} d \mathcal{A}_{t}^{\dagger}\left(\Psi^{*}\right)+U_{t}^{*} d \mathcal{A}_{t}\left(\Phi^{*}\right)+U_{t}^{*} d \mathcal{L}_{t}\left(Z^{*}\right), U_{0}^{*}=\mathbb{1}, t \in[0, T]$,

where $T>0$ is a fixed finite horizon, $F$ is a bounded operator on the system space $\mathcal{H}, \Psi, \Phi$, and $Z$ are of the same form as $D_{-}, D_{+}$, and $D_{1}$ respectively, and $u_{t}$ is of the form $-\Pi U_{t}$ for some positive bounded system operator $\Pi$.

The functional

$$
Q_{\xi, T}(u)=\int_{0}^{T}\left[\left\langle U_{t} \xi, X^{2} U_{t} \xi\right\rangle+\left\langle u_{t} \xi, u_{t} \xi\right\rangle\right] d t-\left\langle u_{T} \xi, U_{T} \xi\right\rangle,
$$

where $X$ is a system space observable, identified with its ampliation $X \otimes I$ to $\mathcal{H} \otimes \Gamma$, is minimized over the set of feedback control processes of the form $u_{t}=-\Pi U_{t}$ by choosing $\Pi$ to be a bounded, positive, self-adjoint system operator satisfying

$$
\begin{aligned}
\Pi F+F^{*} \Pi+(\Phi \mid \Pi \Phi)-\Pi^{2}+X^{2} & =0, \\
\Pi \Psi+\Phi^{*} \Pi+l(\Pi Z) \Phi^{*} & =0, \\
\Pi Z+Z^{*} \Pi+\left(Z^{*} \Pi\right) \circ Z & =0 .
\end{aligned}
$$

The minimum value is $\langle\xi, \Pi \xi\rangle$.

Proof. The proof follows in a way similar to that of Theorem 1 with the use of the square of white noise Itô table of Proposition 2.

THEOREM D Let $X$ be a bounded self-adjoint system operator such that the pair $(i H, X)$ is stabilizable. The quadratic performance functional (5.5) associated with the quantum stochastic flow $\left\{j_{t}(X)=U_{t}^{*} X U_{t}: t \geq 0\right\}$, where $U=\left\{U_{t}: t \geq 0\right\}$ is the solution of (5.1), is minimized by choosing

$$
D_{-}=\sum_{n} D_{-, n} \otimes e_{n}
$$


and

$$
W=\sum_{\alpha, \beta, \gamma} W_{\alpha, \beta, \gamma} \otimes \rho^{+}\left(B^{+\alpha} M^{\beta} B^{-\gamma}\right)
$$

such that

$$
\frac{1}{2}\left(D_{-}^{*} \mid D_{-}^{*}\right)=\left(\frac{1}{2} \sum_{n} D_{-, n} D_{-, n}^{*}\right) \otimes \mathbb{1}=\Pi,
$$

and

$$
\begin{aligned}
{\left[D_{-, n}, D_{-, m}\right] } & =\left[D_{-, n}, D_{-, m}^{*}\right]=0 \\
{\left[D_{-, n}, W_{\alpha, \beta, \gamma}\right] } & =\left[D_{-, n}, W_{\alpha, \beta, \gamma}^{*}\right]=0
\end{aligned}
$$

for all $n, m, \alpha, \beta, \gamma$, which also implies that $\left[D_{-, n}^{*}, W_{\alpha, \beta, \gamma}\right]=\left[D_{-, n}^{*}, W_{\alpha, \beta, \gamma}^{*}\right]=0$, where $\Pi$ is a positive self-adjoint solution of the algebraic Riccati equation

$$
i[H, \Pi]+\Pi^{2}+X^{2}=0 .
$$

Moreover

$$
\min _{D_{-}, W} J_{\xi, T}\left(D_{-}, W\right)=\langle\xi, \Pi \xi\rangle
$$

independently of $T$.

Proof. Looking at (5.1) as (5.6) with $u_{t}=-\frac{1}{2}\left(D_{-}^{*} \mid D_{-}^{*}\right) U_{t}$ i.e

$$
\Pi=\frac{1}{2}\left(D_{-}^{*} \mid D_{-}^{*}\right)=\left(\frac{1}{2} \sum_{n} D_{-, n} D_{-, n}^{*}\right) \otimes \mathbb{1},
$$

$F=i H, \Psi=D_{-}, \Phi=-r(W) D_{-}^{*}$, and $Z=W-I,(5.8)$ is identical to (5.5) and equations (5.9)-(5.11) become

$$
\begin{aligned}
i[\Pi, H]+\left(r(W) D_{-}^{*} \mid \Pi r(W) D_{-}^{*}\right)-\Pi^{2}+X^{2} & =0, \\
\Pi D_{-}-l\left(W^{*}\right) D_{-} \Pi-l(\Pi(W-I)) l\left(W^{*}\right) D_{-} & =0, \\
\Pi(W-I)+\left(W^{*}-I\right) \Pi+\left(\left(W^{*}-I\right) \Pi\right) \circ(W-I) & =0 .
\end{aligned}
$$

Equation (5.21) implies $W^{*} \Pi \circ W=\Pi \Rightarrow W^{*} \Pi \circ W \circ W^{*}=\Pi \circ W^{*} \Rightarrow W^{*} \Pi \circ I=$ $\Pi \circ W^{*} \Rightarrow W^{*} \Pi=\Pi W^{*} \Rightarrow[\Pi, W]=\left[\Pi, W^{*}\right]=0$ and (5.16) follows from (5.14). Similarly, (5.20) implies that $\left[\Pi, D_{-}\right]=0$ from which (5.15) follows. Finally, using the fact that $\left(r(W) D_{-}^{*} \mid r(W) D_{-}^{*}\right)=\left(D_{-}^{*} \mid r\left(W^{*}\right) r(W) D_{-}^{*}\right)=\left(D_{-}^{*} \mid D_{-}^{*}\right),(5.19)$ implies (5.17). 


\section{Bibliography}

[1] L. Accardi, Quantum Kalman filtering. Mathematical system theory. The influence of R.E Kalman, Springer, 1991, pp. 135-143.

[2] L. Accardi, A. Boukas, Unitarity conditions for stochastic differential equations driven by nonlinear quantum noise, Random Operators and Stochastic Equations 10 (1), 1 (2002).

[3] L. Accardi, A. Boukas, Stochastic evolutions driven by non-linear quantum noise, Probability and Mathematical Statistics 22 (1), xxx (2002).

[4] L. Accardi, A. Boukas, Stochastic evolutions driven by non-linear quantum noise II, Russian Journal of Mathematical Physics 8 (4), xxx (2001).

[5] L. Accardi, A. Boukas, Square of white noise unitary evolutions on Bson Fock space, to appear in Proceedings of the 'International Conference on Stochastic Analysis' in honour of Paul Kree, Hammamet, Tunisie, October 22-27, 2001.

[6] L. Accardi, A. Boukas, Unitarity conditions for the renormalized square of white noise, Trends in Contemporary Infinite Dimensional Analysis and Quantum Probability, Natural and Mathematical Sciences Series 3, Italian School of East Asian Studies, Kyoto, Japan, 2000, pp. 7-36.

[7] L. Accardi, A. Boukas, The semi-martingale property of the square of white noise integrators, in: Stochastic Partial Differential Equations and Applications, G. Da Prato and L. Tubaro, eds., Marcel Dekker, Inc., 2002, pp. 1-19.

[8] L. Accardi, A. Boukas, Control of elementary quantum flows, Proceedings of the 5th IFAC symposium on nonlinear control systems, July 4-6, 2001, St. Petersburg, Russia.

[9] L. Accardi, A. Boukas, The unitarity conditions for the square of white noise, to appear in: Infinite Dimensional Anal. Quantum Probab. Related Topics, (2003).

[10] L. Accardi, A. Boukas, Quadratic control of quantum processes, Russian Journal of Mathematical Physics 9 (4), 381 (2002).

[11] L. Accardi, A. Boukas, H. H. Kuo, On the unitarity of stochastic evolutions driven by the square of white noise, Infinite Dimensional Analysis, Quantum Probability, and Related Topics 4 (4), 1 (2001).

[12] L. Accardi, F. Fagnola, J. Quaegebeur, A representation free quantum stochastic calculus, Journal of Functional Analysis 104 (1), 149 (1992).

[13] L. Accardi, U. Franz, M. Skeide, Renormalized squares of white noise and non- Gaussian noises as Levy processes on real Lie algebras, Comm. Math. Phys. 228 (1), 123 (2002).

[14] L. Accardi, T. Hida, H. H Kuo, The Itô table of the square of white noise, Infinite Dimensional Analysis, Quantum Probability, and Related Topics 4, 267 (2001).

[15] L. Accardi, Y. G Lu, I. V Volovich, Quantum theory and its stochastic limit, Springer, 2002.

[16] L. Accardi, Y. G Lu, I. V Volovich, White noise approach to classical and quantum stochastic calculi, Lecture Notes of the Volterra International School of the same title, Trento, Italy, 1999, Volterra Center preprint 375.

[17] L. Accardi, Y. G Lu, I. V Volovich, Non-linear extensions of classical and quantum stochastic calculus and essentially infinite dimensional analysis, Volterra Center preprint 268, 1996.

[18] L. Accardi, N. Obata, Towards a non-linear extension of stochastic calculus, Publications of the Research Institute for Mathematical Sciences, Kyoto, RIMS Kokyuroku 957, Obata N. ed., 1996, pp. 1-15.

[19] L. Accardi, M. Skeide, On the relation of the square of white noise and the finite difference algebra, Infinite Dimensional Analysis, Quantum Probability, and Related Topics 3, 185 (2000).

[20] L. Accardi, M. Skeide, Hilbert module realization of the square of white noise and the finite difference algebra, Volterra Center preprint 384, 1999.

[21] A. Boukas, Quantum stochastic analysis: a non Brownian case, Ph. D Thesis, Southern Illinois University, 1998 
[22] A. Boukas, An example of a quantum exponential process, Monatsh. Math. 112 (3), 209 (1991).

[23] A. Boukas, Stochastic calculus on the finite difference Fock space, Quantum Probability and Related Topics, vol. VI, L. Accardi, ed., World Scientific, 1991, pp. 205-218.

[24] A. Boukas, Linear Quantum Stochastic Control, Quantum Probability and Related Topics, QP -PQ IX, World Scientific Publishing, River Edge, NJ, 1994, pp. 105-111.

[25] A. Boukas, Application of Operator Stochastic Calculus to an Optimal Control problem, Mat. Zametki 53 (5), 48 (1993); translation in Math. Notes 53 (5-6), 489 (1993).

[26] A. Boukas, Operator valued stochastic control in Fock space with applications to noise filtering and orbit tracking, Journal of Probability and Mathematical Statistics 16 Fasc. 2, 221 (1996).

[27] A. Boukas, Stochastic Control of operator-valued processes in Boson Fock space, Russian Journal of Mathematical Physics 4 (2), 139 (1996).

[28] P. J. Feinsilver, Discrete analogues of the Heisenberg-Weyl algebra, Monatsh. Math. 104, 89 (1987).

[29] K. R. Parthasarathy, An introduction to quantum stochastic calculus, Birkhauser Boston Inc., 1992.

[30] A. J. Pritchard, R.F. Curtain, The infinite dimensional Riccati equation, J. Math. Anal. and Appl. 47, xxx (1974). 